
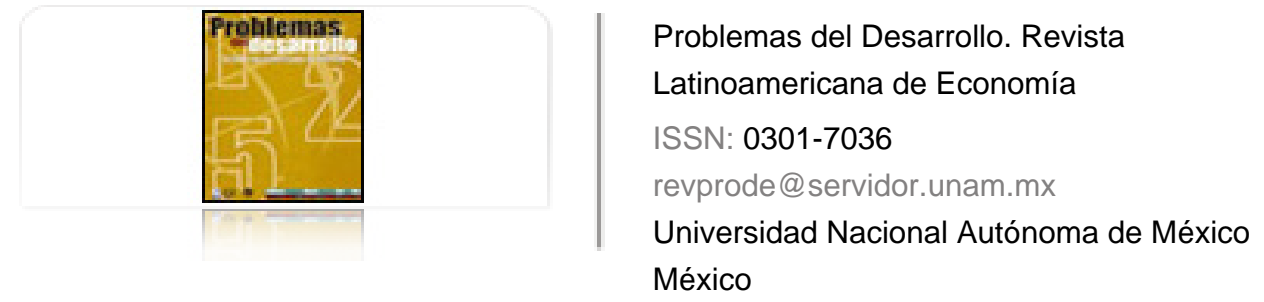

Rivas Aceves, Salvador; Venegas-Martínez, Francisco Participación del gobierno en el desarrollo tecnológico: un modelo de crecimiento endógeno para una economía monetaria

Problemas del Desarrollo. Revista Latinoamericana de Economía, vol. 39, núm. 152, enero-marzo, 2008, pp. 47-68

Universidad Nacional Autónoma de México

Distrito Federal, México

Disponible en: http://www.redalyc.org/articulo.oa?id=11820668004

Cómo citar el artículo

- Número completo

- Más información del artículo

Página de la revista en redalyc.org

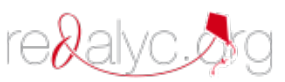

Sistema de Información Científica

Red de Revistas Científicas de América Latina, el Caribe, España y Portugal Proyecto académico sin fines de lucro, desarrollado bajo la iniciativa de acceso abierto 


\title{
PARTICIPACIÓN DEL GOBIERNO EN EL DESARROLLO TECNOLÓGICO: UN MODELO DE CRECIMIENTO ENDÓGENO PARA UNA ECONOMÍA MONETARIA
}

\author{
Salvador Rivas Aceves* \\ Francisco Venegas-Martínez**
}

Fecha de recepción: 9 de octubre de 2007. Fecha de aceptación: 7 de enero de 2008.

\section{Resumen}

En el presente trabajo se desarrolla un modelo de crecimiento endógeno en donde el gobierno interviene en la generación de tecnología. En el marco de una economía monetaria se caracteriza el nivel óptimo de gasto de gobierno para impulsar el nivel tecnológico y, en consecuencia, el crecimiento económico; asimismo se analiza el impacto que dicho gasto tiene sobre el bienestar económico. Por último, se establecen varias recomendaciones en materia de política industrial, orientadas hacia el incremento del producto marginal del capital.

Palabras clave: crecimiento endógeno, gasto de gobierno, cambio tecnológico, dinero, política industrial.

* Investigador del Departamento de Economía de la Universidad Autónoma Metropolitana, Unidad Azcapotzalco, en donde también es profesor. Correo electrónico: rivas.salvador@gmail.com.

** Profesor en la Escuela Superior de Economia del IPN. Correo electronico: fvenegas1111@ yahoo.com.mx. 


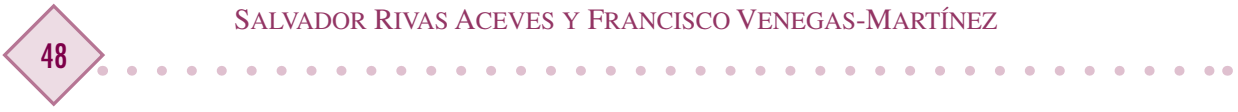

\section{Summary}

This study develops an endogenous growth model in which the government intervenes in the generation of technology. In the framework of a monetary economy the optimum amount of government spending is characterized to promote the technological level, and, as a consequence, economic growth. Meanwhile, it analyzes the impact that this spending has on economic welfare. Finally, some recommendations are made for industrial policy, oriented towards increasing the marginal product of capital.

Key words: endogenous growth, government spending, technological change, money, industrial policy.

\section{Résumé}

Dans ce travail est développé un modèle de croissance endogène où le gouvernement intervient dans la génération de technologie. Dans le cadre d'une économie monétaire, on considère et caractérise le niveau optimum de dépense du gouvernement pour impulser le progrès technologique et, par conséquent, la croissance économique; ainsi même est analysé l'impact que cette dépense a sur le bien être économique. Finalement, diverses recommandations sont faites en matière de politique industrielle, visant à l'accroissement du produit marginal de capital.

Mots clés: croissance endogène, dépense du gouvernement, changement technologique, argent, politique industrielle.

\section{Resumo}

No presente trabalho desenvolve-se um modelo de crescimento endógeno onde o governo intervém na geração de tecnologia. No marco de uma economia monetária caracteriza-se o nível ótimo de gasto de governo para impulsionar o nível tecnológico e, em conseqüência, o crescimento econômico; além disso, analisa-se o impacto que tal gasto tem sobre o bem-estar econômico. Por último, estabelecem-se várias recomendações em matéria de política industrial, orientadas ao incremento do produto marginal do capital.

Palavras-chave: crescimento endógeno, gasto de governo, mudança tecnológico, dinheiro, política industrial. 


\section{Introducción}

D e manera endógena, el análisis del crecimiento económico de largo plazo ha tomado mucha fuerza dentro de la teoría del crecimiento desde los trabajos de Romer $(1986,1990)$ y Lucas $(1988)$, en los cuales se resalta que la endogenidad de los desarrollos teóricos es pertinente. Inicialmente, la introducción del gobierno en la teoría del crecimiento se hizo en función del impacto que éste genera sobre el mismo, por medio de las políticas gubernamentales que logren modificar la tasa de retorno del capital, como en el caso de Jones y Manuelli (1990).

Muchos otros modelos de crecimiento endógeno explican el crecimiento económico en términos del comportamiento del gobierno; véanse, por ejemplo, Barro (1990) que estudia cómo el gasto de gobierno y el tamaño relativo del mismo repercuten en el crecimiento económico; Futagami-Morita-Shibata (1993) amplían el análisis de Barro y muestran que la tasa de crecimiento es la máxima cuando la tasa de ingresos impositivos es igual a la elasticidad del producto respecto al capital público; Venegas-Martínez (1999) que establece que si la deuda pública externa crece a una tasa mayor que la deuda externa privada, entonces existe un tradeoff entre deuda pública externa y crecimiento; y en Venegas-Martínez (2008) que analiza cómo la política fiscal afecta al crecimiento.

En el marco de una economía monetaria, la presente investigación estudia el efecto de la participación del gobierno en el desarrollo de tecnología en un modelo de crecimiento endógeno. A diferencia de los modelos de crecimiento neoclásicos estándar, la utilización de dicho instrumental en este modelo permitirá explicar el efecto de la política gubernamental tecnológica sobre la tasa de crecimiento. El dinero entra en el modelo por dos motivos: para el financiamiento del consumo (Clower, 1967) y como argumento en la función de utilidad (Patinkin, 1956; Sidrauski, 1967).

La organización del trabajo es como sigue: primero se describe el modelo básico de crecimiento endógeno, después se incorpora el comportamiento del gobierno. En el transcurso de los siguientes apartados, en el marco de una economía monetaria, se caracteriza el nivel óptimo de gasto de gobierno para el desarrollo de tecnología y se analiza el impacto de dicho gasto en el bienestar económico. Finalmente se presentan las conclusiones, así como las limitaciones y sugerencias para futuras investigaciones. También se presenta un apéndice con los detalles de los resultados analíticos del problema de maximización de utilidad de los agentes. 


\section{El modelo básico}

En esta sección se describe, brevemente, el modelo básico de crecimiento endógeno a fin de tener un punto de referencia que permita resaltar las diferencias cuantitativas y cualitativas que surjan al modelo propuesto cuando se introduzca el gobierno como agente promotor de la tecnología. Así pues, considere una economía poblada por consumidores con preferencias y dotaciones idénticas, cada uno de los cuales viven para siempre y desean aumentar su satisfacción por un bien genérico de consumo. Suponga que dicha satisfacción está dada por:

$$
U=\int_{0}^{\infty} u\left(c_{t}\right) e^{-\rho t} \mathrm{~d} t
$$

donde $c_{t}$ es consumo per capita y $\rho$ es la tasa subjetiva de descuento; este último parámetro mide qué tan ansioso está un individuo por el consumo presente. En todo el análisis subsiguiente se utilizará el siguiente índice de bienestar:

$$
u\left(c_{t}\right)=\ln c_{t}
$$

Evidentemente, esta especificación cumple con $u^{\prime}\left(c_{t}\right)>0$ y $u^{\prime \prime}\left(c_{t}\right)<0$; es decir, la utilidad marginal es positiva pero decreciente. En otras palabras, el consumo siempre proporciona satisfacción, pero a medida que éste aumenta, la satisfacción que produce va disminuyendo. Se supone que el consumidor es al mismo tiempo productor y la función de producción tiene la forma $y_{t}=f\left(k_{t}\right)$. En lo que sigue, se considera una función de producción del tipo de Harrod-Rebelo (Harrod, 1939; Rebelo, 1991), a saber:

$$
f\left(k_{t}\right)=A k_{t}
$$

donde $A>0$ es el producto marginal del capital. Por tanto, el problema de maximización del agente representativo se puede expresar de la siguiente manera:

$$
\begin{aligned}
& \text { Maximizar } U=\int_{0}^{\infty} \ln c_{t} e^{-\rho t} \mathrm{~d} t \\
& \text { s.a. } \quad \dot{k}_{t}=A k_{t}-c_{t}, \quad k_{0} \text { dado }
\end{aligned}
$$

En este caso, las condiciones de primer orden están dadas por:

$$
\frac{\partial \mathcal{H}}{\partial c_{t}}=0, \quad \frac{\partial \mathcal{H}}{\partial \lambda_{t}}=\dot{k}_{t} \quad \mathrm{y}-\frac{\partial \mathcal{H}}{\partial k_{t}}=\dot{\lambda}_{t}-\lambda_{t} \rho
$$


donde $\mathcal{H}=\ln c_{t}+\lambda_{t}\left(A k_{t}-c_{t}\right)$ es el hamiltoniano. La condición de transversalidad que se tiene que cumplir es:

$$
\lim _{t \rightarrow \infty} k_{t} e^{-A t}=0
$$

En este caso, la trayectoria del consumo tiene la siguiente forma:

$$
c_{t}=\rho k_{0} e^{(A-\rho) t}
$$

Como puede observarse, el consumo depende del parámetro de preferencias $\rho$, del capital inicial $k_{0}$ y del coeficiente de tecnología $A$. Evidentemente, si $A>\rho$, se tiene crecimiento en el consumo. La gráfica 1 muestra el comportamiento del consumo cuando $A>\rho$. Observe también que si $A<\rho$, se tendrá decrecimiento, tal y como lo muestra la gráfica 2.

Gráfica 1

Trayectoria del consumo $(\mathrm{A}>\mathrm{Rho})$

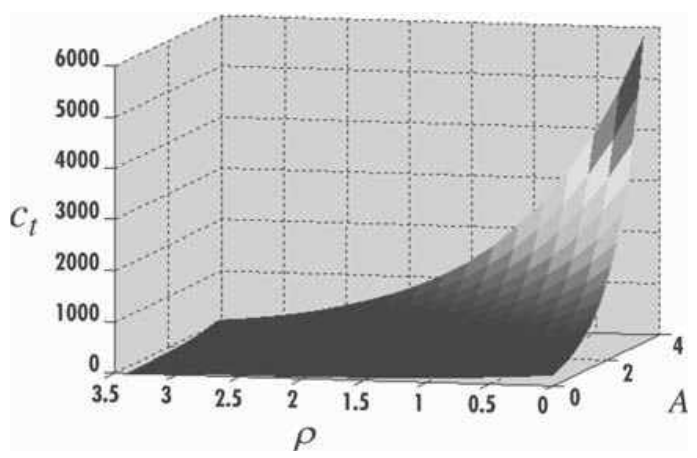

Gráfica 2

Trayectoria del consumo $(\mathrm{A}<\mathrm{Rho})$

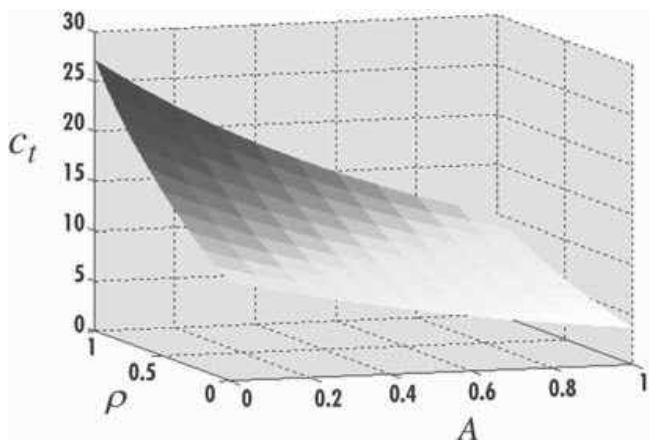


En el modelo básico se puede mostrar que la trayectoria del capital está dada por:

$$
k_{t}=k_{0} e^{(A-\rho) t}
$$

En consecuencia, el capital depende, obviamente, del acervo de capital inicial $k_{0}$ y éste crecerá si $A>\rho$; véase, al respecto, la gráfica 3. Por otro lado, si se cumple que $A<\rho$, entonces se presentará un decrecimiento en el capital; véase ahora la gráfica 4 :

Gráfica 3

Trayectoria del capital $(\mathrm{A}>\mathrm{Rho})$

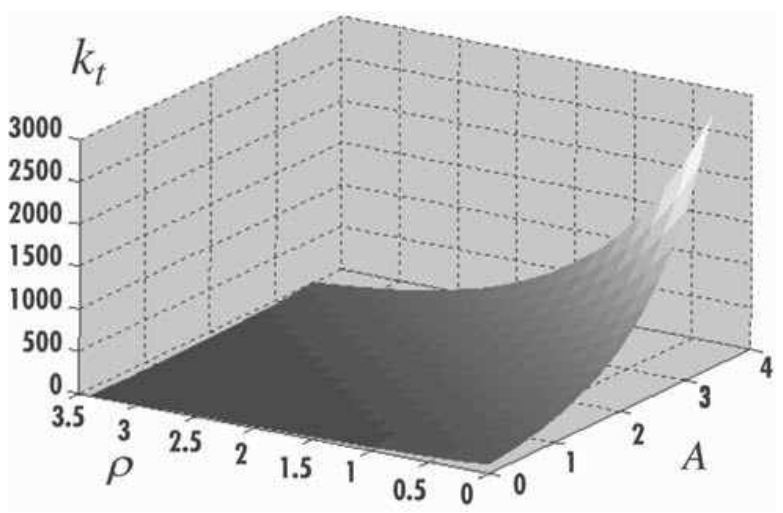

Gráfica 4

Trayectoria del capital $(\mathrm{A}<\mathrm{Rho})$

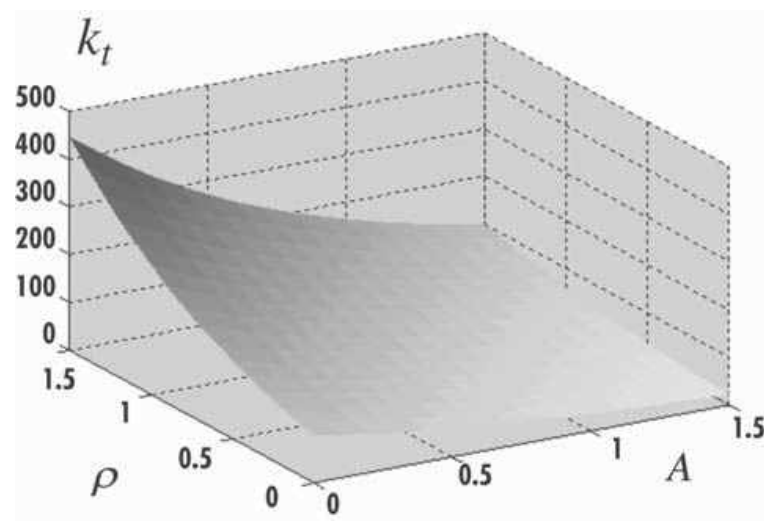


Una vez que se obtienen las dinámicas del consumo $\dot{c}_{t}=c_{t}(A-\rho)$ y del capital $\dot{k}_{t}=k_{t}(A-\rho)$, y en virtud de que la función de producción es de la forma $y_{t}=A k_{t}$, entonces se puede determinar la tasa de crecimiento económico per capita $\gamma$ :

$$
\gamma=\frac{\dot{y}_{t}}{y_{t}}=\frac{\dot{c}_{t}}{c_{t}}=\frac{\dot{k}_{t}}{k_{t}}=A-\rho
$$

Por tanto, la tasa de crecimiento en todos los sectores de la economía depende del parámetro de la tecnología y de la tasa subjetiva de descuento. Si $A>\rho$ todos los sectores crecen, mientras que si $A<\rho$, entonces decrecen. Observe que el crecimiento es igual en todos los sectores; en otras palabras, el crecimiento es balanceado. En consecuencia, países con coeficientes tecnológicos altos crecen a tasas mayores.

\section{El gobierno como agente multiplicador de la tecnología}

Ahora se supone que el gobierno interviene en el desarrollo de tecnología afectando la productividad del capital, de tal forma que la función de producción toma la siguiente manera:

$$
y_{t}=A g k_{t}
$$

en donde $g>0$ es el gasto que el gobierno realizará como inversión en la tecnología. En consecuencia, el problema de maximización del consumidor tiene ahora la siguiente forma:

$$
\begin{aligned}
& \text { Maximizar } U=\int_{0}^{\infty} \ln c_{t} e^{-\rho t} \mathrm{~d} t \\
& \text { s.a. } \dot{k}_{t}=A g k_{t}-c_{t}, \quad k_{0} \text { dado }
\end{aligned}
$$

Con respecto al problema de crecimiento endógeno básico, las condiciones de primer orden y la condición de transversalidad se convierten en:

$$
\frac{\partial \mathcal{H}}{\partial c_{t}}=0, \frac{\partial \mathcal{H}}{\partial \lambda_{t}}=\dot{k}_{t}, \quad-\frac{\partial \mathcal{H}}{\partial k_{t}}=\dot{\lambda}_{t}-\lambda_{t} \rho
$$

y

$$
\lim _{t \rightarrow \infty} k_{t} e^{-(A g) t}=0
$$

donde $\mathcal{H}=\ln c_{t}+\lambda_{t}\left(g A k_{t}-c_{t}\right)$ es el hamiltoniano. En este caso, la trayectoria del consumo satisface:

$$
c_{t}=\rho k_{0} e^{(A g-\rho) t}
$$


A partir del resultado anterior se desprende que el consumo está en función del parámetro de preferencias $\rho$, del capital inicial $k_{0}$ y del gasto de gobierno $g$. Un aumento en las últimas dos variables incrementará el nivel de consumo. Por otro lado, se tiene que si $A g>\rho$, con $g>1$, entonces el consumo crecerá a un mayor ritmo que en el caso en donde no interviene el gobierno, tal y como lo muestra la gráfica 5. En caso contrario, es decir, si $A g<\rho$, entonces el consumo decrecerá. Este efecto se puede apreciar en la gráfica 6.

Gráfica 5

Trayectoria del consumo $(\mathrm{Ag}>\mathrm{Rho})$

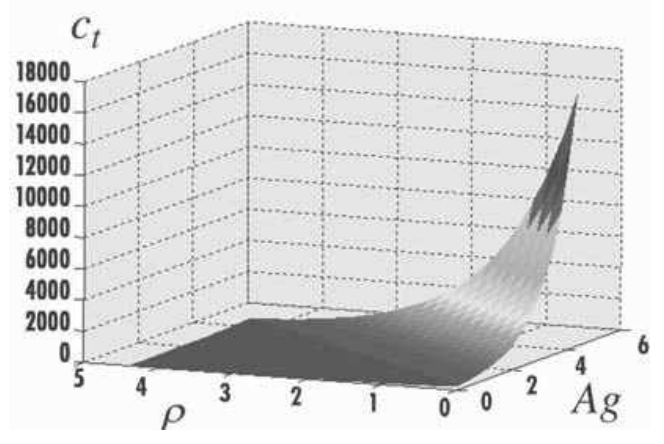

Gráfica 6

Trayectoria del consumo $(\mathbf{A g}<\mathbf{R h o})$

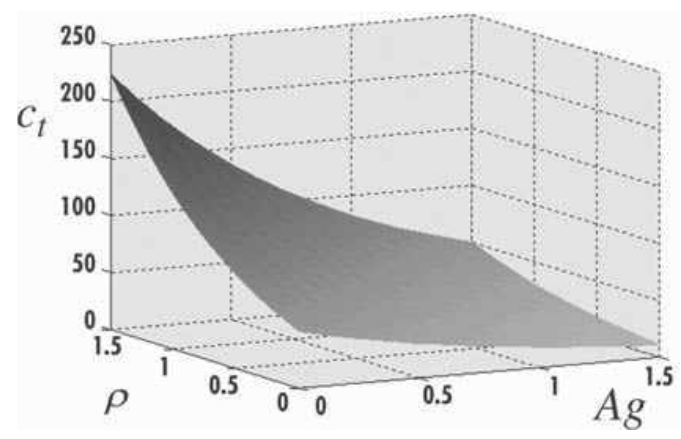

Ahora bien, es deseable que $g>1$, ya que sólo así $A g>A$. De esta forma, si el gobierno invierte en tecnología, entonces éste puede influir en el crecimiento económico. Por otro lado, se tiene que la trayectoria del capital es igual a:

$$
k_{t}=k_{0} e^{(A g-\rho) t}
$$


A partir de esta ecuación se observa, una vez más, que si $A g>\rho$, entonces el capital crecerá, véase la gráfica 7; lo contrario sucederá si $A g<\rho$, véase la gráfica 8 .

Gráfica 7

Trayectoria del capital $(\mathrm{Ag}>\mathrm{Rho})$

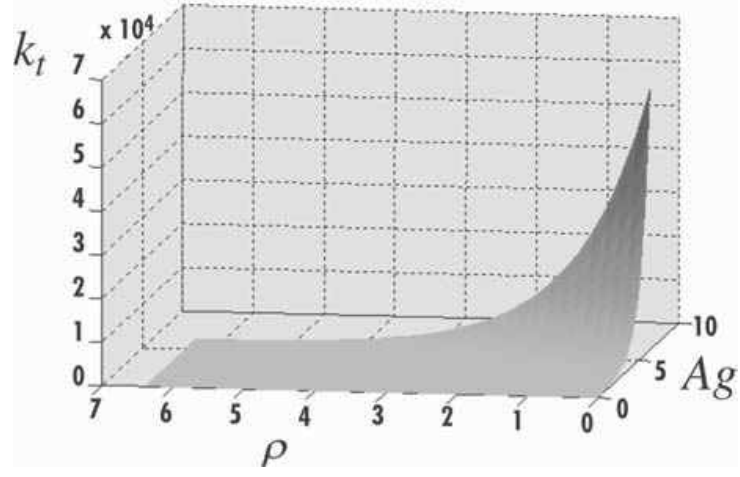

Gráfica 8

Trayectoria del capital $(\mathrm{Ag}<\mathrm{Rho})$

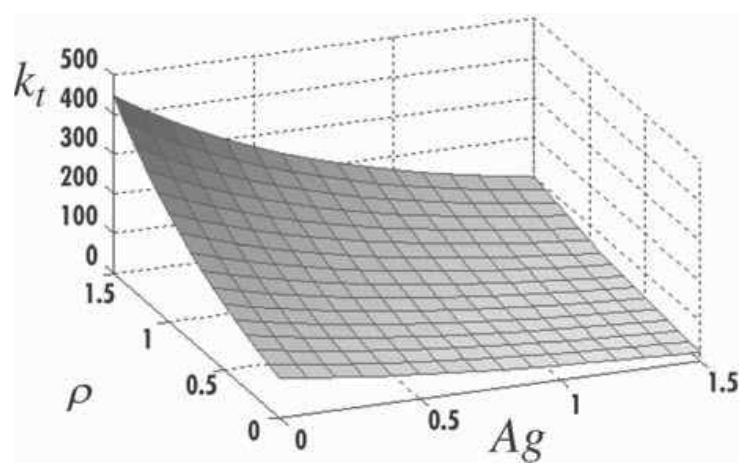

Como puede observarse, las trayectorias del consumo y del capital dependen de la tasa subjetiva de descuento, de la tecnología y del gasto de gobierno de tal forma que $\dot{c}_{t}=c_{t}(A g-\rho)$ y $\dot{k}_{t}=k_{t}(A g-\rho)$, entonces la tasa de crecimiento per capita estará dada por:

$$
\gamma=\frac{\dot{y}_{t}}{y_{t}}=\frac{\dot{c}_{t}}{c_{t}}=\frac{\dot{k}_{t}}{k_{t}}=A g-\rho
$$




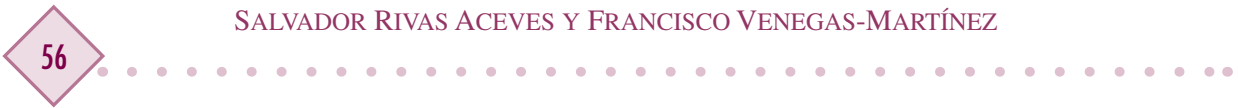

En conclusión, la tasa de crecimiento en todos los sectores de la economía depende de la diferencia $\mathrm{Ag}-\rho$. Por tanto, si $\mathrm{Ag}>\rho$, todos los sectores crecen, mientras que decrecen si $A g<\rho$, entonces todos los sectores decrecen. Observe que, de nuevo, el crecimiento es balanceado.

A continuación se elige el nivel óptimo de gasto de gobierno y se evalúa cuál es su impacto en el bienestar de los agentes. Para ello se debe obtener, primero, la función del bienestar económico o utilidad indirecta, $\mathcal{W}$, que resulta de la sustitución del nivel óptimo de consumo en la función directa de utilidad. En consecuencia

$$
W=\int_{0}^{\infty} \ln \left(\rho k_{0} e^{(A g-\rho) t}\right) e^{-\rho t} \mathrm{~d} t
$$

Equivalentemente

$$
W=\frac{\ln \left(\rho k_{0}\right)}{\rho}+\frac{A g-\rho}{\rho^{2}}
$$

Por tanto, el bienestar económico depende del coeficiente tecnológico disponible $A$, del impacto del gobierno $g$, de las preferencias del individuo $\rho$ y del capital inicial $k_{0}$, es decir, $\mathcal{W}=\mathcal{W}\left(A, g, k_{0}, \rho\right)$. El siguiente análisis de estática comparativa permitirá conocer bajo qué condiciones se puede mejorar el bienestar. Al derivar la función del bienestar con respecto de $g$, se obtiene que:

$$
\frac{\partial W}{\partial g}=\frac{A}{\rho^{2}}>0
$$

Debido a que esta cantidad es positiva, entonces un aumento en el gasto de gobierno, como inversión en tecnología, aumenta el bienestar. De esta manera, el gobierno no sólo participa en la generación de tecnología, sino que con ello incrementa, a su vez, el bienestar de los agentes. Ahora bien, si se deriva la función con respecto de $A$ se tiene que:

$$
\frac{\partial W}{\partial A}=\frac{g}{\rho^{2}}>0
$$

Dado que esta cantidad es positiva, un aumento en la tecnología por medio del parámetro $A$, elevará el bienestar. Evidentemente, se espera que en países con un mayor desarrollo tecnológico el nivel de bienestar económico sea más alto que en naciones tecnológicamente rezagadas. Por otro lado, la derivada de la función con respecto de $k_{0}$ es:

$$
\frac{\partial W}{\partial k_{0}}=\frac{1}{\rho k_{0}}>0
$$


Esta cantidad es mayor a cero, lo cual indica que un aumento en el capital inicial aumentará el bienestar. Por último, si se deriva la función bienestar con respecto de $\rho$, se tiene que:

$$
\frac{\partial W}{\partial \rho}=-\frac{2}{\rho^{3}}<0
$$

Es decir, un incremento en la tasa subjetiva de descuento, lo cual se traduce en una mayor ansiedad por parte del individuo para consumir en el presente, deteriora el bienestar.

En conclusión, el bienestar crecerá conforme se tenga un aumento en el capital inicial, o en el gasto de gobierno, o si se da un incremento en el nivel de tecnología mediante el coeficiente $A$. Por otro lado, el bienestar disminuirá si aumenta la tasa subjetiva de descuento.

\section{Gobierno como multiplicador de la tecnología y dinero como motivo transacción}

En este apartado se introduce el dinero en la economía mediante el supuesto de que el agente representativo mantiene saldos reales con el objetivo de financiar su consumo, debido a que "el dinero compra bienes, los bienes compran dinero, pero los bienes no compran bienes", véase Blanchard (1998). De esta manera, el dinero se incorpora bajo la forma del motivo transacción. Sean $m_{t}=M_{t} / P_{t}$ los saldos reales per capita y $\pi$ el costo por la tenencia de saldos reales, entonces la restricción presupuestal del individuo toma la siguiente forma:

$$
\dot{m}_{t}+\dot{k}_{t}=A g k_{t}-c_{t}-\pi m_{t}
$$

Si se supone también que el total de la riqueza real del individuo está dada por la suma de su capital y sus saldos monetarios, es decir, $a_{t}=m_{t}+k_{t}$, entonces

$$
\dot{m}_{t}+\dot{k}_{t}=A g k_{t}+A g m_{t}-A g m_{t}-c_{t}-\pi m_{t}
$$

Equivalentemente

$$
\dot{a}_{t}=A g a_{t}-c_{t}-(A g+\pi) m_{t}
$$

A continuación se incorpora una restricción del tipo cash-in-advance, propuesta por Clower (1967), a saber:

$$
m_{\mathrm{t}}=\int_{t}^{t+\alpha} c_{\mathrm{s}} \mathrm{d} s
$$


donde $\alpha$ es el tiempo que el dinero debe ser mantenido para financiar el consumo; observe que:

$$
m_{\mathrm{t}}=\int_{t}^{t+\alpha} c_{\mathrm{s}} \mathrm{d} s \approx \alpha c_{t}+o(\alpha)
$$

En lo que sigue, se supondrá que el término del error $o(\alpha)$ es despreciable, en cuyo caso $m_{t}=\alpha c_{t}$, es decir, se requieren saldos reales para financiar consumo durante el tiempo $\alpha$. En consecuencia, al sustituir dicha condición en la restricción presupuestal, se obtiene:

$$
\dot{a}_{t}=A g a_{t}-[1+(\alpha A g+\alpha \pi)] c_{t}
$$

Por tanto, el nuevo problema de maximización de utilidad del individuo está dado por:

$$
\begin{aligned}
& \text { Maximizar } U=\int_{0}^{\infty} \ln c_{t} e^{-\rho t} \mathrm{~d} t \\
& \text { s.a. } \quad \dot{a}_{t}=A g a_{t}-[1+(\alpha A g+\alpha \pi)] c_{t}, \quad a_{0} \text { dado }
\end{aligned}
$$

Las condiciones de primer orden y la condición de transversalidad son, respectivamente:

$$
\begin{gathered}
\frac{\partial \mathcal{H}}{\partial c_{t}}=0, \frac{\partial \mathcal{H}}{\partial \lambda_{t}}=\dot{a}_{t}, \quad-\frac{\partial \mathcal{H}}{\partial a_{t}}=\dot{\lambda}_{t}-\lambda_{t} \rho \\
\lim _{t \rightarrow \infty} a_{t} e^{-(A g) t}=0
\end{gathered}
$$

donde $\mathcal{H}=\ln c_{t}+\lambda_{t}\left(A g a_{t}-[1+\alpha(A g+\pi)] c_{t}\right)$ es el hamiltoniano. En este caso, la trayectoria del consumo resulta ser positiva, como lo muestra la gráfica 9:

$$
c_{t}=\frac{\rho a_{0}}{[1+\alpha(A g+\pi)]} e^{(A g-\rho) t}
$$

Gráfica 9

Trayectoria del consumo $(\mathbf{A g}>\mathbf{R h o})$

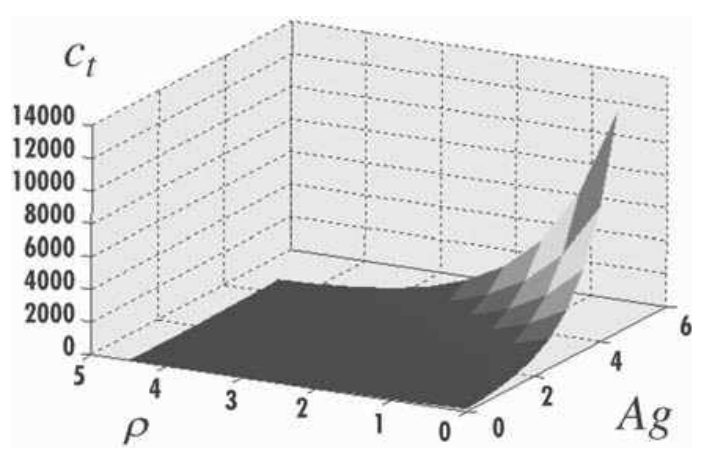


Por lo anterior, el consumo depende de la riqueza inicial, del parámetro de preferencias, del nivel de tecnología, del gasto de gobierno, de la proporción de saldos reales que se mantenga y de la inflación. Una vez más se cumple que si $A g>\rho$, el consumo aumenta y disminuye si $\mathrm{Ag}<\rho$. Por otro lado, la trayectoria de la riqueza es conducida por la siguiente ecuación:

$$
a_{t}=a_{0} e^{(A g-\rho) t}
$$

En consecuencia, la trayectoria de la riqueza depende del nivel de riqueza inicial y crecerá o disminuirá a la tasa $A g-\rho$. Dada la ecuación anterior, si $A g>\rho$, el comportamiento de la riqueza se muestra en la gráfica 10.

Gráfica 10

Trayectoria de la riqueza $(\mathbf{A g}>\mathbf{R h o})$

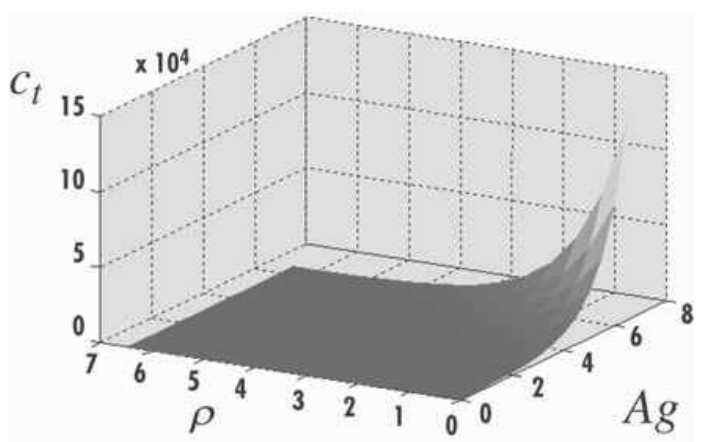

Se concluye ahora que las trayectorias del consumo y de riqueza dependen de la tasa subjetiva de descuento, de la tecnología y del gasto de gobierno por medio de $\dot{c}_{t}=c_{t}(A g-\rho) \quad$ y $\quad \dot{a}_{t}=a_{t}(A g-\rho) \mathrm{y}$, entonces

$$
\frac{\dot{c}_{t}}{c_{t}}=\frac{\dot{a}_{t}}{a_{t}}=A g-\rho
$$

Al igual que en el modelo anterior, si $A g>\rho$ todos los sectores crecen, mientras que decrecen si $A g<\rho$, y el crecimiento sigue siendo balanceado. Dicho crecimiento no depende de la tasa de crecimiento de los saldos reales por lo que el dinero es neutral. 
Para elegir el nivel óptimo de gasto que el gobierno debe realizar para mejorar el bienestar, una vez que el individuo ha decidido sobre sus niveles de consumo y riqueza, se obtiene la función del bienestar mediante la sustitución del consumo óptimo en la función de utilidad, lo cual conduce a:

$$
W=\int_{0}^{\infty} \ln \left\{\frac{\rho a_{0}}{[1+(\alpha A g+\alpha \pi)]} e^{(A g-\rho) t}\right\} e^{-\rho t} \mathrm{~d} t
$$

Equivalentemente

$$
\mathcal{W}=\ln \left\{\frac{a_{0}}{[1+(\alpha A g+\alpha \pi)]}\right\}+\frac{A g-\rho}{\rho^{2}}
$$

Para encontrar el nivel óptimo de $g$, se deriva la función $\mathcal{W}$ con respecto de $g$ y se iguala a cero. Por tanto, se tiene:

$$
\frac{\partial W}{\partial g}=-\frac{a_{0} \alpha A}{g}+\frac{A}{\rho^{2}}=0
$$

Si se despeja $g$ se obtiene:

$$
g=a_{0} \alpha \rho^{2}
$$

Por tanto, el nivel óptimo del gasto de gobierno está en función de la riqueza inicial de los individuos $a_{0}$, de la proporción de dinero que éstos utilicen para financiar su consumo $\alpha$ y del parámetro de preferencias $\rho$. Por tanto, como el gobierno conoce la riqueza inicial de los individuos, la proporción de saldos reales que éstos mantienen y sus preferencias, entonces sabe exactamente cuál es el gasto óptimo de inversión en tecnología que permitirá tener una mayor tasa de crecimiento; evidentemente, se requiere $g>1$. Además, dicha tasa será igual en todos los sectores, lo que generará un crecimiento balanceado.

\section{Gobierno como multiplicador de la tecnología y dinero como reserva de valor}

Si ahora se hace el supuesto de que al agente representativo le genera satisfacción mantener saldos reales en su poder (por sus servicios de liquidez), entonces el dinero se debe introducir en la función de utilidad (Sidrausky, 1967). La función de utilidad es estrictamente cóncava y ambas mercancías son no inferiores, y como ahora ya no se tiene una restricción cash-in-advance, entonces la restricción presupuestal del 
individuo queda como se había planteado previamente. Por lo anterior, el problema de maximización del individuo representativo es ahora:

$$
\begin{aligned}
& \text { Maximizar } U=\int_{0}^{\infty}\left(\ln c_{t}+\beta \ln m_{t}\right) e^{-\rho t} \mathrm{~d} t \\
& \text { s.a. } \dot{a}_{t}=A g a_{t}-(A g+\pi) m_{t}-c_{t}, \\
& \text { con } a_{0} \text { dado, } \quad \beta>0 .
\end{aligned}
$$

Las condiciones de primer orden junto con la condición de transversalidad ahora son:

$$
\begin{gathered}
\frac{\partial \mathcal{H}}{\partial c_{t}}=0 \quad \frac{\partial \mathcal{H}}{\partial m_{t}}=0 \quad \frac{\partial \mathcal{H}}{\partial \lambda_{t}}=\dot{a}_{t} \quad-\frac{\partial \mathcal{H}}{\partial a_{t}}=\dot{\lambda}_{t}-\lambda_{t} \rho \\
\lim _{t \rightarrow \infty} a_{t} e^{-(A g) t}=0
\end{gathered}
$$

donde $\mathcal{H}=\ln c_{t}+\lambda_{t}\left(A g a_{t}-(A g+\pi) m_{t}-c_{t}\right)$ es el hamiltoniano. Las trayectorias de consumo y de los saldos reales son respectivamente:

$\mathrm{y}$

$$
c_{t}=\frac{\rho a_{0}}{\beta+1} e^{(A g-\rho) t}
$$

$$
m_{t}=\frac{\rho a_{o}-1}{A g+\pi} e^{(A g-\rho) t}
$$

Por tanto, el consumo depende de la riqueza inicial y de la tasa subjetiva de descuento. Los saldos reales dependen de la riqueza inicial, del nivel de tecnología, del gasto de gobierno y de la inflación; ambos crecerán a la tasa $A g-\rho$ cuando $A g>\rho$, y decrecerán si $A g<\rho$. Se requiere suponer que $\rho a_{0}>1$ a fin de garantizar que $\rho a_{0}>1$. La representación del comportamiento de las demandas de consumo y saldos reales se muestran, respectivamente, en las gráficas 11 y 12 .

Gráfica 11

Trayectoria del consumo $(\mathbf{A g}>\mathbf{R h o})$

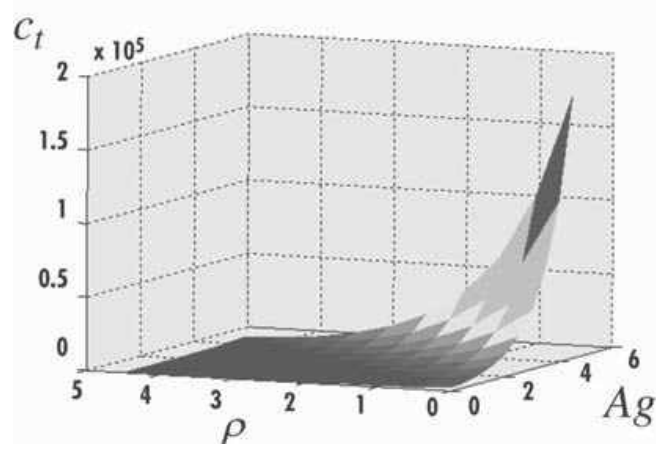




\section{Gráfica 12}

Trayectoria de los saldos reales $(\mathrm{Ag}>\mathrm{Rho})$

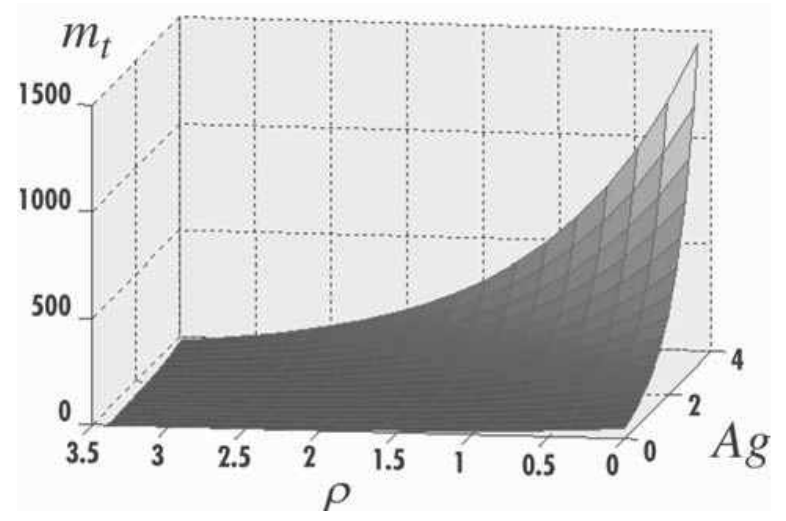

En este caso, se encuentra que las trayectorias del consumo y de la demanda de saldos reales están dadas por:

$$
\dot{c}_{t}=c_{t}(A g-\rho) \quad \text { y } \quad \dot{m}_{t}=m_{t}(A g-\rho)
$$

Finalmente, las trayectorias del consumo y de los saldos reales dependen de la tasa subjetiva de descuento, de la tecnología y del gasto de gobierno. En conclusión

$$
\frac{\dot{c}_{t}}{c_{t}}=\frac{\dot{m}_{t}}{m_{t}}=A g-\rho
$$

Los resultados señalan que el dinero es neutral, lo que concuerda con los obtenidos en estado estacionario por Sidrausky (1967). En este documento el estado estacionario no se analiza por lo que la no neutralidad encontrada por Reis (2001) no se puede refutar. Por otro lado, Fischer (1979) encontró que la tasa de crecimiento del dinero afecta a la tasa de acumulación de capital y por tanto a la tasa de crecimiento económico, en consecuencia el dinero es no neutral. Aunque es importante señalar que la diferencia entre el modelo de Sidrausky y el de Fischer es que en el primero se suponen expectativas adaptativas y en el segundo expectativas racionales (Asako 1983).

Una vez que el individuo ha decidido cuáles son sus niveles óptimos de consumo y saldos reales, se necesita elegir cuál es el nivel óptimo de gasto de gobierno, por lo 
que se debe obtener la función de bienestar sustituyendo dichos óptimos en la función de utilidad, en este caso se encuentra que:

$$
W=\int_{0}^{\infty} \ln \left[\frac{\rho a_{0}}{\beta+1} e^{(A g-\rho) t}\right]+\beta \ln \left(\frac{\rho a_{0}-1}{A g+\pi} e^{(A g-\rho) t}\right) e^{-\rho t} \mathrm{~d} t
$$

Equivalentemente

$$
W=\ln \left(\frac{a_{0}}{(\beta+1)}\right)+\frac{(A g-\rho)}{\rho^{2}}+\beta \ln \left(\frac{\rho a_{0}-1}{(A g+\pi) \rho}\right)+\frac{(A g-\rho)}{\rho^{2}}
$$

Para obtener el gasto óptimo del gobierno se deriva la función $W$ con respecto de $g$ y se iguala a cero:

$$
\frac{\partial W}{\partial g}=\frac{A}{\rho^{2}}-\frac{\beta}{A g \rho}+\frac{A}{\rho^{2}}=0
$$

Una vez que se despeja $g$, se tiene que el nivel óptimo del gasto de gobierno es:

$$
g=\frac{\beta \rho}{2 A^{2}}
$$

Se aclara entonces que el nivel de gasto óptimo depende de parámetro de preferencias $\rho$, del nivel de la tecnología $A$ y de la utilidad que le proporciona la tenencia de saldos reales al individuo $\beta$.

\section{Conclusiones}

Se ha desarrollado un modelo de agente representativo con vida infinita en donde se incorpora la participación del gobierno mediante la inversión en tecnología y emisión de dinero. Uno de los principales resultados obtenidos es que cuando el gobierno participa en la generación de tecnología, con ello incrementa también el bienestar de los agentes. Otro resultado relevante es que la tasa de crecimiento económico no depende de la tasa de expansión monetaria, es decir, el dinero es neutral.

Debido a que el crecimiento - el cual es balanceado- depende del gasto de gobierno, se concluye que el gobierno tiene fuertes incentivos para participar en la generación de tecnología, pues con ello también afecta a los otros sectores. Se encontró además que el gasto de gobierno óptimo que genera el mayor bienestar posible depende de la riqueza inicial y de los parámetros de preferencias de los individuos, así como de la cantidad de dinero que los individuos tengan en su poder. 
Por otro lado, al incorporar el dinero en la función de utilidad al agente, se obtuvo una vez más que la tasa de crecimiento de la economía no es afectada por la tasa de expansión monetaria, lo que significa que existe neutralidad del dinero. En este caso, el gasto óptimo que debe realizar el gobierno para generar un crecimiento económico - y, por ende, una mejora en el bienestar- está en función de la tecnología, de las preferencias y de la utilidad del dinero por sus servicios de liquidez. En cualquiera de las versiones que se utilizaron para incorporar el dinero se tiene que la tasa de crecimiento de la economía es independiente de la tasa de expansión monetaria, por tanto se comprueba que el dinero es neutral.

A pesar de lo anterior, las principales limitaciones que se encuentran en esta investigación consisten en suponer que el gobierno sólo interviene en la generación de tecnología, que la economía es cerrada y que las variables son deterministas. Se debería extender el análisis a una economía en donde el gobierno, además de generar tecnología, pueda participar en otras actividades; la economía debe ser abierta y, por ende, con comercio exterior; así como la incorporación de variables estocásticas para un modelado más realista del comportamiento de variables económicas y financieras fundamentales.

\section{Apéndice}

Derivación de la fórmula (32)

Del problema de optimización planteado en (29), las condiciones de primer orden son:

$$
\begin{gathered}
\frac{\partial \mathcal{H}}{\partial c_{t}}=\frac{1}{c_{t}}-\lambda_{t}[1+(\alpha A g+\alpha \pi)]=0 \\
\frac{\partial \mathcal{H}}{\partial \lambda_{t}}=A g a_{t}-[1+(\alpha A g+\alpha \pi)] c_{t}=\dot{a}_{t} \\
-\frac{\partial \mathcal{H}}{\partial a_{t}}=-\lambda_{t}(A g)=\dot{\lambda}_{\bar{t}} \quad \lambda_{t} \rho
\end{gathered}
$$

Después de despejar el consumo de (A.1), se obtiene:

$$
c_{t}=\frac{1}{\lambda_{t}[1+(\alpha A g+\alpha \pi)]}
$$

Al factorizar de (A.3) $\lambda_{t}$ y, posteriormente, despejar $\dot{\lambda}_{t}$ se sigue que

$$
\dot{\lambda}_{t}=\lambda_{t}(\rho-A g)
$$


Si $\dot{x}_{t}=\alpha x_{t}$ la solución de la ecuación diferencial es de la forma $x_{t}=x_{0} e^{\alpha t}$, entonces las soluciones de las ecuaciones diferenciales (A.2) y (A.5) son respectivamente:

$$
\begin{gathered}
a_{0}=\int_{0}^{\infty}[1+(\alpha A g+\alpha \pi)] c_{t} e^{-(A g) t} \mathrm{~d} t \\
\lambda_{t}=\lambda_{0} e^{(\rho-A g) t}
\end{gathered}
$$

Si se sustituye (A.7) en (A.2), se obtiene:

$$
c_{t}=\frac{1}{\lambda_{0}[1+(\alpha A g+\alpha \pi)]} e^{-(\rho-A g) t}
$$

Al sustituir la ecuación (A.8) en (A.6), se tiene:

$$
a_{0}=\int_{0}^{\infty} \frac{[1+(\alpha A g+\alpha \pi)]}{[1+(\alpha A g+\alpha \pi)] \lambda_{0}} e^{-(\rho-A g) t} e^{-(A g) t} \mathrm{~d} t
$$

Después de simplificar se produce la siguiente ecuación:

$$
a_{0}=\frac{1}{\lambda_{0} \rho} \int_{0}^{\infty} \rho e^{-\rho t} \mathrm{~d} t
$$

Observe que $\int_{0}^{\infty} \mu e^{-\mu x} \mathrm{~d} x=1 \quad " \mu>0$, entonces

$$
\frac{1}{\lambda_{0}}=\rho a_{0}
$$

De esta manera, cuando se sustituye la ecuación (A.11) en (A.8), se encuentra la trayectoria del consumo:

$$
c_{t}=\frac{\rho a_{0}}{[1+(\alpha A g+\alpha \pi)]} e^{(A g-\rho) t}
$$

Derivación de la ecuación (33)

Si se sustituye la ecuación (A.12) en (A.2), se obtiene:

$$
\dot{a}_{t}=(A g) a_{t}-\rho a_{0} e^{(A g-\rho) t}
$$

Se sabe que si $\dot{x}_{t}=\alpha x_{t}+f(t)$, entonces la solución es de la forma $x_{t}=x_{0} e^{\alpha t}+e^{\alpha t} \int_{0}^{t} f(s) e^{-\alpha s} \mathrm{~d} s$. Por tanto, se obtiene que:

$$
a_{t}=a_{0} e^{(A g) t}-e^{(A g) t} \int_{0}^{t} \rho a_{0} e^{(A g-\rho) s} e^{-(A g) s} \mathrm{~d} s
$$


Al resolver, la ecuación anterior, se sigue que:

$$
a_{t}=a_{0} e^{(A g-\rho) t}
$$

Derivación de las ecuaciones (42) y (43)

Las condiciones de primer orden del problema planteado en (39) son:

$$
\begin{gathered}
\frac{\partial \mathcal{H}}{\partial c_{t}}=\frac{1}{c_{t}}-\lambda_{t}=0 \\
\frac{\partial \mathcal{H}}{\partial m_{t}}=\frac{\beta}{m_{t}}-\lambda_{t}(A g+\pi)=0 \\
\frac{\partial \mathcal{H}}{\partial \lambda_{t}}=A g a_{t}-(A g+\pi) m_{t}-c_{t}=\dot{a}_{t} \\
-\frac{\partial \mathcal{H}}{\partial a_{t}}=-\lambda_{t}(A g)=\dot{\lambda}_{t}-\lambda_{t} \rho
\end{gathered}
$$

Por tanto, al despejar el consumo de (A.16) y los saldos reales de (A.17), se tiene respectivamente que:

y

$$
\begin{gathered}
c_{t}=\frac{1}{\lambda_{t}} \\
m_{t}=\frac{\beta}{\lambda_{t}(A g+\pi)}
\end{gathered}
$$

Después de despejar $\dot{\lambda}_{t}$ de (A.19), resulta:

$$
\dot{\lambda}_{t}=\lambda_{t}(\rho-A g)
$$

Una vez determinadas las ecuaciones diferenciales (A.18) y (A.22), si se supone que $\lim _{t \rightarrow \infty} a_{t} e^{-(A g) t}=0$, se tienen, respectivamente,

$$
a_{0}=\int_{0}^{\infty}(A g+\pi) m_{t}+c_{t} e^{-(A g) t} \mathrm{~d} t
$$

y

$$
\lambda_{t}=\lambda_{0} e^{(\rho-A g) t}
$$

Si se sustituye la ecuación (A.24) tanto en (A.20) como en (A.21), se obtienen:

$$
c_{t}=\frac{1}{\lambda_{0} e^{(\rho-A g) t}}
$$

$$
m_{t}=\frac{\beta}{\lambda_{0} e^{(\rho-A g) t}(A g+\pi)}
$$


Si se sustituyen ahora las ecuaciones (A.25) y (A.26) en la ecuación (A.23), resulta que:

$$
a_{0}=\left(\frac{\beta+1}{\lambda_{0} \rho}\right) \int_{0}^{\infty} \rho e^{-\rho t} \mathrm{~d} t
$$

En consecuencia, debido a que $\int_{0}^{\infty} \mu e^{-\mu x} \mathrm{~d} x=1 \quad \mu>0$, si se despejan $\frac{1}{\lambda_{0}}$ y $\beta$, se llega a que:

$\mathrm{y}$

$$
\frac{1}{\lambda_{0}}=\frac{\rho a_{0}}{\beta+1}
$$

$$
\beta=\lambda_{0} \rho a_{0}-1
$$

Por último, para encontrar la trayectoria del consumo se sustituye la ecuación (A.28) en (A.25), y para hallar la trayectoria de los saldos reales se sustituye la ecuación (A.29) en (A.26), con lo que se obtienen, respectivamente:

$$
c_{t}=\frac{\rho a_{0}}{\beta+1} e^{(A g-\rho) t}
$$

$\mathrm{y}$

$$
m_{t}=\frac{\rho a_{o}-1}{A g+\pi} e^{(A g-\rho) t}
$$

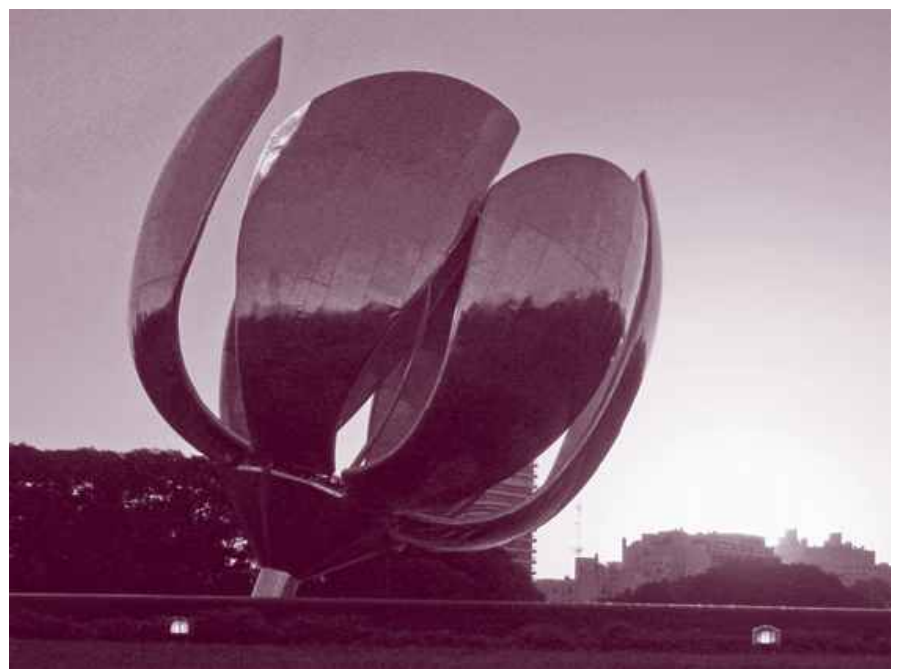

La flor I. Buenos Aires, verano 2005-2006. PAI 


\section{Bibliografía}

Azako, Kazumi, "The Utility Function and the Superneutrality of Money on the Transition Path", en Econometrica, vol. 51, núm. 5, septiembre de 1983, pp. 1593-1596.

Barro, Robert, "Government Spending in a Simple Model of Endogenous Growth", en The Journal of Political Economy, vol. 98, núm. 5, octubre de 1990, pp. S103-S125.

Blanchard, Oliver y Stanley Fisher, Lectures of Macroeconomics, Massachusetts \& London, The MIT Press, 1998, p. 165.

Clower, Robert, "A Reconsideration of the Microfoundations of Monetary Theory", en Western Economic Journal, vol. 6, diciembre de 1967, pp. 1-8.

Fischer, Stanley, "Capital Accumulation on the Transition Path in a Monetary Optimizing Model", en Econometrica, vol. 47, núm. 6, noviembre de 1979, pp. 1433-1439.

Futagami, Koichi, Morita, Yuichi, y Akihisa Shibata, "Dynamic Analysis of a Endogenous Growth Model with Public Capital", The Scandinavian Journal of Economics, vol. 95, núm. 4, 1993, pp. 607-625.

Harrod, Roy, "An Essay in Dynamic Theory", en Economic Journal, vol. 49, núm. 193, marzo de 1939, pp. 14-33.

Jones, Larry y Rodolfo Manuelli, "A Convex Model of Equilibrium Growth: Theory and Policy Implications", The Journal of Political Economy, vol. 98, núm. 5, Part 1, 1990, pp. 1008-1038.

Lucas, Robert, "On the Mechanics of Economic Development", Journal of Monetary Economics, 22, 1988, pp. 3-42.
Patinkin, Don, Money, Interest and Prices, Massachusetts, The MIT Press, 1956.

Rebelo, Sergio, "Long Run Policy Analysis and Long Run Growth", en The Journal of Political Economy, vol. 99, núm. 3, junio de 1991, pp. 500-521.

Reis, Ricardo, The Analytics of Monetary NonNeutrality in the Sidrauski Model, Princeton, Princeton University Prees, 2001, pp. 1-8.

Romer, Paul, "Increasing Returns and LongRun Growth", The Journal of Political Economy, vol. 94, núm. 5, 1986, pp. 1002-1037.

,"Endogenous Technological Change", The Journal of Political Economy, vol. 98, núm. 5, part 2: The Problem of Development: A Conference of the Institute for the Study of Free Enterprise System, 1990, pp. S71-S102.

Sidrauski, Miguel, "Rational Choices and Patterns of Growth in a Monetary Economy", en American Economic Review, vol. 57, núm. 2, mayo de 1967, pp. 534-544.

Venegas-Martínez, Francisco, "Crecimiento endógeno, dinero, impuestos y deuda externa", en Investigación Económica, vol. 59, núm. 229, 1999, pp.15-36.

"Un modelo estocástico de equilibrio macroeconómico: acumulación de capital, inflación y política fiscal”, en Investigación Económica (por aparecer, 2008). 